\title{
On the Capacity Scalings of the Multiple Antenna Group-Broadcast Systems
}

\author{
Amir F. Dana ${ }^{1}$, Tareq Y. Al-Naffouri ${ }^{2}$, and Babak Hassibi ${ }^{1}$ \\ ${ }^{1}$ Electrical Engineering Dept., California Institute of Technology, Pasadena, CA \\ ${ }^{2}$ Electrical Engineering Dept., King Fahd University of Petroleum and Minerals, Saudi Arabia
}

\begin{abstract}
In this paper, we consider a multi-user system called the group-broadcast system. In this scenario the users are divided into different groups. Users in each group are interested in a common information independent from that of other groups. Such a situation occurs for example in digital audio and video broadcast systems where the users are divided into various groups according to the shows they are interested in. The paper first obtains upper and lower bounds for the sum rate capacity. Then it looks at system capacity for the large number of users regime and fixed number of antennas. Finally, the case when the number of users and antennas grow simultaneously is studied. It is shown that in order to achieve a constant rate per user the number of transmit antennas should scale at least logarithmically in the number of users.
\end{abstract}

\section{INTRODUCTION}

Given the increasing emergence of high data rate application for wireless networks, there has a great deal of research on the fundamental capabilities and practical design of multiuser systems in recent years. Examples of these systems are broadcast channels and multiple access channels that model the downlink and uplink of cellular systems respectively [1]. The MIMO Gaussian broadcast channel (GBC) resembles downlink communication in a cellular system, where the base station (possibly equipped with multiple transmit antennas) is to convey independent messages (e.g., voice calls) to different users. Much work has been devoted to find the capacity region of MIMO GBC. The capacity region question was recently settled by a technique called dirty paper coding (DPC). Specifically, [2] and [3] have shown that DPC is capable of achieving the maximum possible sum-rate capacity. Subsequently, [4] showed that DPC is able to achieve any point in the capacity region. Different practical schemes are also devised that can deliver much of this capacity with lower complexity compared to DPC [4]-[9].

The broadcast scenario considered above assumes that the various users are interested in independent streams of data. More common is the situation that one group of users would be interested in one stream of data, another group with another stream, and so on. An example where this might occur is digital audio and video broadcast where there is a limited number of shows and users are classified according to the shows they are interested in [10], [11], [12]. We will refer to such a scenario as the group-broadcast systems. Analyzing the sum-capacity of these systems is the main topic of this paper. We should remark that the definition of group-broadcast systems include the broadcast systems described above and the multicast systems. Simply, in a broadcast system each group consists of only one user. In the multicast scenario, there is only one group of user present in the system. All the users demand the same information. The capacity of multicast system with Gaussian channels has been recently considered in [13], [15] and [16]. [15] finds the capacity scaling for large number of users. In [16] scaling order of various techniques in a multicast scenario with multiple antennas at the base station is analyzed.

In this paper, we consider the multiple group in the large number of users and antennas regime. The paper is organized as follows. In the first part of the paper, we consider the large number of users $(n)$ case and obtain upper bounds (in Section III) and lower bounds (in Section IV). In the rest of the paper, we consider the scaling for the large number of antennas $(M)$ regime. We do so for $n=\beta M$ (Section $\mathrm{V}$ ) and for $n=e^{M}$ (Section VI).

\section{System Model AND BACKGRound}

\section{A. System Model}

Consider a group-broadcast system with a base station equipped with $M$ antennas and $n$ users each equipped with a single receive antenna. The users are partitioned into $K$ groups. For simplicity of exposition, we will assume that the number of users in each group is $\frac{n}{K}$. The different number of users case can be treated similarly. The received signal at the $i \mathrm{TtH}$ user is given by

$$
y_{i}=h_{i}^{*} s+n_{i}
$$

where $s$ is the transmitted signal, and $n_{i} \sim C N\left(0, I_{M}\right)$ is the additive Gaussian noise. We consider a block fading model in this paper with coherent interval of $T . h_{i}$ is the channel matrix between the base station and user $i$ and is distributed according to $C N\left(0, I_{M}\right)$ and is assumed to be i.i.d over different users ${ }^{1}$. We further assume that the transmitter is subject to an average power constraint $P$, i.e., $\mathrm{E}\left[\mathrm{ss}^{*}\right] \leq P$. We denote the capacity region of the group-broadcast system under power constraint $P$, by $\mathcal{C}_{g b}(P)$. The sum-capacity point is also the maximum of the sum of the rates in the capacity region and is denoted by $C_{g b}$.

We should mention here that if the transmitter had one antenna only, then the capacity region of the group-broadcast

\footnotetext{
${ }^{1}$ The results of this paper can be easily extended to the case where there is correlation between different antennas.
} 
system is known and is equivalent to the capacity region of a broadcast channel. For in this case, all channels involved would be single input single output. Thus, to transmit to the $k$-th group of users, one simply needs to take care of the user with the weakest link, i.e. the link for which $\left|h_{i, k}\right| \leq\left|h_{j, k}\right|$ for all $j$. Such ordering of users, however, is not possible in the multiple antenna case and the problem becomes more challenging.

\section{B. A Result in Extreme Value Theory}

Here, we will mention a result concerning the scaling behavior of the minimum of a large number of i.i.d random variable. We will use this result throughout this paper. Let $X_{1}, X_{2}, \cdots, X_{n}$ be iid nonnegative random variables with $\mathrm{CDF} F(x)$, and characteristic function $\phi(x)$. We would like to find the scaling law of the minimum of these random variables, $X_{\min }(n)=\left\{X_{1}, X_{2}, \cdots, X_{n}\right\}$. We can state the following lemma regarding the asymptotic behavior of $X_{\min }(n)$. The proof of this lemma is omitted due to space limitations and can be found in [18].

Lemma 1: Let $i_{0}$ be the first non-zero derivative of $F(x)$ at zero, i.e., $F^{\left(i_{0}\right)}(0) \neq 0$ and $F^{(j)}(0)=0$ for all $j<i_{0}$. Then $n^{\frac{1}{i_{0}}} X_{\min }(n)$ converges in distribution to random variable $Y$ with $\mathrm{CDF}$ of

$$
F_{Y}(y)=1-\exp \left(-\frac{F^{\left(i_{0}\right)}(0)}{i_{0} !} y^{i_{0}}\right) .
$$

Furthermore, we can find $F^{\left(i_{0}\right)}(0)$ using the initial value theorem as follows

$$
\lim _{x \rightarrow 0} F^{\left(i_{0}\right)}(x)=\lim _{s \rightarrow \infty} s^{i_{0}} \phi(s) .
$$

a) Example: Let's find the scaling laws for $\min _{i}\left\|h_{i}\right\|^{2}$ when $h_{i}$ are iid $C N(0, R)$. The pdf and CDF of $\left\|h_{i}\right\|^{2}$ will both have different forms depending on the whether some of the eigenvalues $\lambda_{l}$ of $R$ are the same or different. On the other hand, the characteristic function takes one form and is given by

$$
\phi(s)=\prod_{l=1}^{M} \frac{1}{1+\lambda_{l} s}
$$

From this, it is easy to see that

$$
\lim _{s \rightarrow \infty} s^{i} \phi(s)=F^{(i)}(0)=0 \text { for } i<M
$$

and that

$$
\lim s^{M} \phi(s)=F^{(M)}(0)=\frac{1}{\prod_{l=1}^{M}} \frac{1}{\lambda_{l}}=\frac{1}{\operatorname{det}(R)}
$$

We thus conclude that

$$
\mathrm{E} \min _{i}\left\|h_{i}\right\|^{2} \text { scales as } \operatorname{det}(R)^{\frac{1}{M}} \frac{1}{n^{\frac{1}{M}}}
$$

\section{UPPER BOUNDS}

\section{A. An Upper Bound Using the MAC-BC Duality}

We can use the MAC-BC duality [17] to obtain an upper bound for the sum-capacity of group-broadcast systems. Specifically, the sum-capacity $c_{g b}$ is upper-bounded by the sum-capacity of a broadcast channel with $K$ users, chosen one from each group. More specifically, let $i_{1}, \ldots, i_{k}$ refer to the users selected from group $1, \ldots, k$ respectively. Then we have

$$
C_{g b} \leq \mathrm{E} \max _{b_{k}, \sum b_{k} \leq P} \log \operatorname{det}\left(I+\sum_{k=1}^{K} h_{i_{k}} b_{k} h_{i_{k}}^{*}\right),
$$

where the right hand side is the sum-capacity of the broadcast channel (written in terms of the dual MAC). Since, this rate has to appeal to user selections, we have

$$
C_{g b} \leq \mathrm{E} \min _{i_{1}} \cdots \min _{i_{K}} \max _{b_{k}: \sum b_{k} \leq P} \log \operatorname{det}\left(I+\sum_{k=1}^{K} h_{i_{k}} b_{k} h_{i_{k}}^{*}\right),
$$

where $i_{l}$ is chosen from group $l$ for $1 \leq l \leq K$. Now, to get rid of the determinant in (2), we use the arithmetic-mean geometric-mean (AM-GM) inequality $\operatorname{det}(A) \leq\left(\frac{\operatorname{tr}(A)}{M}\right)^{M}$ to write

$$
\begin{aligned}
C_{g b} & \leq M \log \left(1+\min _{i_{1}} \cdots \min _{i_{K}} \max _{b_{k}} \frac{1}{M} \sum_{k=1}^{K} b_{k}\left\|h_{i}\right\|^{2}\right) \\
& =M \log \left(1+\frac{P}{M} \min _{i_{1}} \cdots \min _{i_{K}} \max _{k}\left\{\left\|h_{i_{1}}\right\|^{2}, \cdots,\left\|h_{i_{K}}\right\|^{2}\right\}\right) \\
& =M \log \left(1+\frac{P}{M} \max _{k} \min _{i_{1}} \cdots \min _{i_{K}}\left\{\left\|h_{i_{1}}\right\|^{2}, \cdots,\left\|h_{i_{K}}\right\|^{2}\right\}\right) \\
& =M \log \left(1+\frac{P}{M} C_{M} \frac{K^{\frac{1}{M}}}{n^{\frac{1}{M}}}\right)
\end{aligned}
$$

where the last line is a direct application of Lemma 1 for random vectors $h_{i}$ and $C_{M}=\frac{\Gamma\left(\frac{1}{M}\right)(M !)^{\frac{1}{M}}}{M}$. Alternatively, and with the aid of the relationship $\operatorname{det}\left(I+A A^{*}\right)=\operatorname{det}\left(I+A^{*} A\right)$, we can show that

$$
C \leq K \log \left(1+\frac{P}{K} C_{M} \frac{K^{\frac{1}{M}}}{n^{\frac{1}{M}}}\right)
$$

From (4) and (5), we conclude that

$$
C=\min \{M, K\} \log \left(1+\frac{P}{\min \{M, K\}} C_{M} \frac{K^{\frac{1}{M}}}{n^{\frac{1}{M}}}\right)
$$

Using the approximation that for small $x, \log (1+x) \simeq x$, we can write

$$
C \leq P C_{M} \frac{K \frac{1}{M}}{n^{\frac{1}{M}}}
$$




\section{B. An Alternative Upper Bound}

Another alternative upper bound is obtained by determining the maximum rate possible assuming there is one group only

$$
\begin{aligned}
\max _{\operatorname{Tr}(B) \leq P} \min _{h_{i}} \log \left(1+h_{i}^{*} B h_{i}\right) & =\log \left(1+\min _{h_{i}} h_{i}^{*} B h_{i}\right) \\
& =\log \left(1+\frac{P}{M} \frac{C_{M}}{n^{\frac{1}{M}}}\right) \\
& \simeq \frac{P}{M} \frac{C_{M}}{n^{\frac{1}{M}}}
\end{aligned}
$$

Thus, for $K$ such groups, the sum rate can not exceed $K$ times the single group case

$$
C \leq K \frac{P}{M} \frac{C_{M}}{n^{\frac{1}{M}}}
$$

Combining this with (7) yields

$$
C \leq \min \left\{1, \frac{K}{M}\right\} P \frac{C_{M}}{n \frac{1}{M}}
$$

\section{LOWER BOUND}

Having obtained an upper bound, we now quantify how various scheduling techniques behave for large number of users. This would give us an idea about the achievable rates. In what follows we consider the following scheduling schemes

- Time sharing

- Scheduling by treating interference as noise

\section{A. Time Sharing}

In this scheduling we assume that the base station time shares in transmission to different groups. More specifically the transmitter allocates $\frac{1}{K}$ of the time to transmit to each group. Clearly this technique provides a lower bound on $C_{g b}$ and we have

$$
\begin{aligned}
C_{g b} & \geq \frac{1}{K} \max _{\operatorname{Tr}(B)=P} \sum_{k=1}^{K} \min _{h_{i_{k}}} \log \operatorname{det}\left(1+h_{i_{k}}^{*} B h_{i_{k}}\right) \\
& =\frac{1}{K} \max _{\operatorname{Tr}(B)=P} \sum_{k=1}^{K} \log \left(1+\min _{h_{i_{k}}} h_{i_{k}}^{*} B h_{i_{k}}\right)
\end{aligned}
$$

We now relax the problem further by setting $B=\frac{P}{M} I$, from which we conclude that

$$
C_{g b} \geq \log \left(1+\frac{P}{M} C_{M} \frac{K^{\frac{1}{M}}}{n^{\frac{1}{M}}}\right)
$$

or using the approximation $\log (1+x)=x$,

$$
C_{g b} \geq \frac{P}{M} C_{M} \frac{K \frac{1}{M}}{n \frac{1}{M}}
$$

\section{B. Treating Interference as Noise}

The other extreme would be to allow all groups to transmit simultaneously. Each group would then ignore signals that are meant for the other groups, treating them as additive noise. Assuming that the covariance matrix of the transmitted signal intended for group $j$ is $B_{j}$ for $j=1, \ldots, K$ with $\operatorname{Tr} \sum B_{j} \leq$ $P$,the rate that the 1 st group achieved with this strategy would be

$$
R_{1}=\min _{i_{1}} \log \left(\frac{h_{i_{1}}^{*} B_{1} h_{i_{1}}}{1+h_{i_{1}}^{*} \sum_{k=2}^{K} B_{k} h_{i_{1}}}\right)
$$

Now, relax the problem further by assuming equal isotropic covariances for all user groups, i.e. set

$$
B_{k}=\frac{1}{K} \frac{P}{M} I
$$

then

$$
\begin{aligned}
R_{i} & =\log \min _{i_{1}} \frac{\frac{1}{K} \frac{P}{M}\left\|h_{i_{1}}\right\|^{2}}{1+\frac{K-1}{K} \frac{P}{M}\left\|h_{i_{1}}\right\|^{2}} \\
& =\log \min _{i_{1}} \frac{1}{K-1}\left(1-\frac{1}{1+\frac{K-1}{K} \frac{P}{M}\left\|h_{i_{1}}\right\|^{2}}\right) \\
& =\log \frac{1}{K-1}\left(1-\frac{1}{1+\frac{K-1}{K} \frac{P}{M} \min _{i_{1}}\left\|h_{i_{1}}\right\|^{2}}\right) \\
& =\log \left(\frac{\frac{1}{K} \frac{P}{M} \min _{i_{1}}\left\|h_{i_{1}}\right\|^{2}}{1+\frac{K-1}{K} \frac{P}{M} \min _{i_{1}}\left\|h_{i_{1}}\right\|^{2}}\right) \\
& =\log \left(\frac{\frac{1}{K} \frac{P}{M} \frac{C_{M}}{n \frac{1}{M}}}{1+\frac{K-1}{K} \frac{P}{M} \frac{C_{M}}{n \frac{1}{M}}}\right) \\
& \simeq \frac{1}{K} \frac{P}{M} \frac{C_{M}}{n \frac{1}{M}}
\end{aligned}
$$

Thus, the sum rate for $K$ such user groups is upper bounded according to

$$
C_{g b} \geq R_{\text {Interf }} \geq \frac{P}{M} \frac{C_{M}}{n \frac{1}{M}}
$$

From the bounds obtained in this section and the previous section, we conclude the following theorem:

Theorem 1: Consider a group broadcast system with $K$ groups, $n$ users and $M$ antennas at the base station and the assumptions of Section II. Assume that $K, M$ are fixed and $n$ grows. Then the sum-capacity $C_{g b}$ of this system scales as

$$
C_{g b}=\alpha P \frac{C_{M}}{n^{\frac{1}{M}}}
$$

where

$$
\frac{1}{M} \leq \alpha \leq \min \left\{1, \frac{K}{M}\right\} .
$$

The result of this theorem is an unfortunate result as it shows that the sum-rate decreases with the number of users. To counter this, we increase the resources (i.e., number of antennas $M$ ). In the rest of this paper, we can study the scaling of group broadcast capacity with the number of antennas for 1) $\frac{M}{n}=\beta$ and 2) $M=\log n$. 
V. SCALING WITH $M$ AND $n, \frac{M}{n}=\beta$

Here we consider the scaling of the upper and lower bounds when the both the number of users and antennas grow to infinity while their ratio remains constant $\frac{M}{n}=\beta$. To this end, notice first that both the upper and the lower bounds depend on the value of $\min _{h_{i}} \frac{\left\|h_{i}\right\|^{2}}{M}$ and so we need to evaluate the scaling of this quantity as $n, M \rightarrow \infty$. To do this, consider the matrix

$$
\Psi=\left[\begin{array}{c}
h_{1}^{*} \\
h_{2}^{*} \\
\vdots \\
h_{n}^{*}
\end{array}\right]\left[\begin{array}{llll}
h_{1} & h_{2} & \cdots & h_{n}
\end{array}\right]
$$

Note that $\operatorname{diag}(\Psi)=\left[\begin{array}{llll}\left\|h_{1}\right\|^{2} & \left\|h_{2}\right\|^{2} & \ldots & \left\|h_{n}\right\|^{2}\end{array}\right]^{T}$. Note also that

$$
\lambda_{\min }\left(\frac{\Psi}{M}\right) \leq \min _{i} \frac{\left\|h_{i}\right\|^{2}}{M} \leq \lambda_{\max }\left(\frac{\Psi}{M}\right) \leq \max _{i} \frac{\left\|h_{i}\right\|^{2}}{M}
$$

Moreover as $n, M \rightarrow \infty$ with $\frac{M}{n}=\beta$, the eigenvalues of $\frac{\Psi}{M}$ become uniformly distributed in the range $\left[(1-\sqrt{\beta})^{2},(1+\right.$ $\left.\sqrt{\beta})^{2}\right]$. We can thus write

$$
(1-\sqrt{\beta})^{2} \leq \lim _{n, M \rightarrow \infty} \min _{i} \frac{\left\|h_{i}\right\|^{2}}{M} \leq(1+\sqrt{\beta})^{2}
$$

This allows us to get a lower bound on capacity which is obtained using time-sharing

$$
\begin{aligned}
& C \geq K \max _{B \geq 0} \operatorname{Tr}(B) \leq P \\
& \frac{1}{K} \log \left(1+\min _{i} h_{i}^{*} B h_{i}\right) \\
& C \geq \log \left(1+P \min _{i} \frac{\left\|h_{i}\right\|^{2}}{M}\right)
\end{aligned}
$$

i.e.,

$$
C \geq \log \left(1+P(1-\sqrt{\beta})^{2}\right)
$$

We obtain the upper bound through another matrix construction. Our starting point is the bound (see Subsection III-B)

$$
\begin{aligned}
C & \leq K \max _{B \geq 0} \operatorname{Tr}(B) \leq P \\
& =K \log \left(1+\min _{i} h_{i}^{*} B h_{i}\right) \\
& \left.\max _{B \geq 0} \operatorname{mr}_{\operatorname{Tr}(B) \leq P} \min _{i} h_{i}^{*} B h_{i}\right)
\end{aligned}
$$

We need an upper bound for $\max _{B>0} \operatorname{Tr}(B)<P \min _{i} h_{i}^{*} B h_{i}$. To do so, we replace the minimization over the $h_{i}$ 's with the sum average (as done in [16])

$$
\begin{aligned}
\max _{B \geq 0} \min _{\operatorname{Tr}(B) \leq P} h_{i}^{*} B h_{i} & \leq \frac{1}{n} \max _{B \geq 0} \sum_{\operatorname{Tr}(B) \leq P}^{n} \sum_{i=1}^{n} B h_{i} \\
& =\frac{1}{n} \max _{B \geq 0} \sum_{\operatorname{Tr}(B) \leq P}^{n} \operatorname{Tr}\left(B h_{i} h_{i}^{*}\right) \\
& =\frac{1}{n} \max _{B \geq 0} \operatorname{Tr}(B) \leq P \\
& \operatorname{Tr}\left(B \sum_{i=1}^{n} h_{i} h_{i}^{*}\right) \\
& =\frac{1}{n} \max _{\operatorname{Tr}(B) \leq P} \operatorname{Tr}\left(B H H^{*}\right) \\
& =\frac{1}{n} P \lambda_{\max }\left(H H^{*}\right)
\end{aligned}
$$

Where $H=\left[\begin{array}{llll}h_{1} & h_{2} & \cdots & h_{n}\end{array}\right]$. Now, as $n, M \rightarrow \infty$ with $\frac{M}{n}=\beta$, the eigenvalues of $\frac{H_{i} H_{i}^{*}}{n}$ will be confined to the range $\left[\left(1-\frac{1}{\sqrt{\beta}}\right)^{2},\left(1+\frac{1}{\sqrt{\beta}}\right)^{2}\right]$. We can thus obtain the following upper bound on capacity

$$
C \leq K \log \left(1+P\left(1+\frac{1}{\sqrt{\beta}}\right)^{2}\right)
$$

Thus, if we allow the number of antennas to grow linearly with the number of users, we can guarantee a constant sum rate. But is it still possible to do so without straining the resources as much?

\section{SCALING With $M$ AND $n, M=\log n$}

In this section, we will look at the behavior of the sumcapacity when $M$ grows logarithmically with $n$. As we will see, this growth is fast enough to guarantee constant rate per user in the system. For this we need to study the behavior of $\min _{i} \frac{\left\|h_{i}\right\|^{2}}{M}$. Note that since $M$ is growing with $n$ we can not simply use the result of Lemma 1 . We will use Chernoff bound instead. Using Chernoff bound for $Y=\frac{\left\|h_{i}\right\|^{2}}{M}$ we have

$$
P(Y \leq 1-\epsilon) \leq e^{\nu(1-\epsilon)} \mathrm{E}\left[e^{-\nu Y}\right],
$$

for any $\nu \geq 0$. Computing the above expectation gives

$$
P\left(\frac{\left\|h_{i}\right\|^{2}}{M} \leq 1-\epsilon\right)=e^{\nu(1-\epsilon)} \frac{1}{\left(1+\frac{\nu}{M}\right)^{M}},
$$

For any $\nu>0$. We can tighten the upper bound by choosing the optimum $\nu$, which, upon setting the first derivative to zero, turns out to be

$$
\nu=M \frac{\epsilon}{1-\epsilon}>0
$$

and the bound reads

$$
\begin{aligned}
P\left(\frac{\left\|h_{i}\right\|^{2}}{M} \leq 1-\epsilon\right) & \leq e^{M \epsilon}(1-\epsilon)^{M} \\
& =e^{M(\epsilon+\log (1-\epsilon))}
\end{aligned}
$$

We can use this to bound the probability $P\left(\min _{i} \frac{\left\|h_{i}\right\|^{2}}{M} \leq 1-\epsilon\right)$

$$
\begin{aligned}
P\left(\min _{i} \frac{\left\|h_{i}\right\|^{2}}{M} \leq 1-\epsilon\right) & =1-\left(1-P\left(\frac{\left\|h_{i}\right\|^{2}}{M} \leq 1-\epsilon\right)\right)^{n} \\
& \leq 1-\left(1-e^{M(\epsilon+\log (1-\epsilon))}\right)^{n} \\
& =1-\left(1-n^{\epsilon+\log (1-\epsilon)}\right)^{n}
\end{aligned}
$$

(14) here the last line follows from the fact that $M=\log n$. For the above probability to vanish as $n$ grows, we require that

$$
\epsilon+\log (1-\epsilon)<-1
$$

Let $\epsilon_{l}$ be the infimum of the set

$$
\begin{aligned}
& \text { i.e., } \epsilon_{l} \text { satisfies } \epsilon_{l}+\log \left(1-\epsilon_{l}\right)=-1 \text { or } \epsilon_{l} \simeq .8414 \text {. Then, } \\
& \qquad \lim _{n \rightarrow \infty} P\left(\min _{i} \frac{\left\|h_{i}\right\|^{2}}{M} \geq 1-\epsilon_{l}\right)=1
\end{aligned}
$$


We can similarly obtain an upper bound for $\min _{i} \frac{\left\|h_{i}\right\|^{2}}{M}$. Employing Chernof bound again, it is easy to show that for $\nu \geq 0$

$$
P\left(\frac{\left\|h_{i}\right\|^{2}}{M} \geq 1+\epsilon\right) \leq e^{-\nu(1+\epsilon)} \frac{1}{\left(1-\frac{\nu}{M}\right)^{M}}
$$

Moreover, the upper bound is tightest for

$$
\nu=M \frac{\epsilon}{1+\epsilon}
$$

We thus have

$$
\begin{aligned}
P\left(\frac{\left\|h_{i}\right\|^{2}}{M} \geq 1+\epsilon\right) & \leq e^{-M \epsilon}(1+\epsilon)^{M} \\
& =e^{M(-\epsilon+\log (1+\epsilon))}
\end{aligned}
$$

or

$$
P\left(\min _{i} \frac{\left\|h_{i}\right\|^{2}}{M} \geq 1+\epsilon\right) \leq\left(n^{(-\epsilon+\log (1+\epsilon))}\right)^{n}
$$

where we used the fact that $n=\log M$. This probability vanishes provided that $-\epsilon+\log (1+\epsilon)<0$ and the infimum for which this is true is $\epsilon_{u}=0$. We can thus write

$$
\lim _{n \rightarrow \infty} P\left(\min _{i} \frac{\left\|h_{i}\right\|^{2}}{M} \leq 1\right)=1
$$

From (18) and (19), we see that

$$
\lim _{n \rightarrow \infty} P\left(\min _{i} \frac{\left\|h_{i}\right\|^{2}}{M} \in\left[1-\epsilon_{l}, 1\right]\right)=1
$$

\section{A. Bound on the Sum-rate Capacity $(M=\log n)$}

We are now ready to derive the lower bound for the sum rate capacity which we obtain through time sharing. Specifically, we have

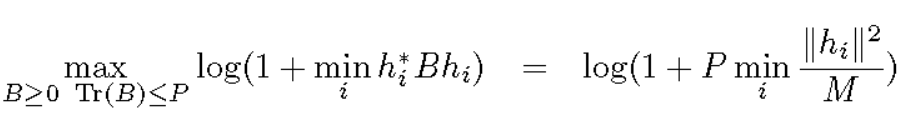

where the second inequality follows by setting $B=\frac{P}{M} I$. Or with $M=\log n$ and as $n \rightarrow \infty$

$$
C \geq \log (1+P \mathcal{H})
$$

where $\mathcal{H} \in\left[1-\epsilon_{l}, 1\right]$. This lower bound shows that a growth of $M=\log n$ will guarantee a constant capacity because for $M=\beta n$ the sum rate is upper bounded by a constant. We have summarized the arguments in this Section in the following theorem.

Theorem 2: Consider a group broadcast system with $K$ groups, $n$ users and $M=\log n$ transmit antennas. Then the sum-capacity in such a system scales like constant and therefore, a constant capacity per user (stream) is achievable in the system.

\section{CONCLUSION}

In this paper, we studied scaling of multi-group broadcast for large number of users. Specifically, we obtained upper and lower bounds for the sum-rate capacity in the large number of users regime. We showed that the sum rate capacity scales as $\alpha P \frac{C_{M}}{n \frac{1}{M}}$. This is an unfortunate result as it shows that the capacity decreases with the number of users. To go around this, we study scaling of the group broadcast capacity with the number of users and antennas. Specifically, we showed that if we set $M=\log n$, we can guarantee a constant rate for each user in spite of the increase in the number of users.

\section{ACKNOWLEDGEMENT}

Tareq Al-Naffouri would like to thank Mihailo Stojnic for fruitful discussions.

\section{REFERENCES}

[1] T. M. Cover and J. A. Thomas, Elements of information theory, New York: Wiley-Interscience, 1991.

[2] S. Vishwanath, N. Jindal, and A. Goldsmith, "Duality, achievable rates and sum rate capacity of Gaussian MIMO broadcast channel," IEEE Trans. Inform., vol. 49, no. 10, pp. 2658-2668, 2003.

[3] P. Viswanath and D. N. Tse, "Sum capacity of the vector Gaussian broadcast channel and downlink-uplink duality," IEEE Trans. Inform., vol. 49, no. 8, pp. 1912-1921, Aug. 2003.

[4] H. Weingarten, Y. Steinberg, and S. Shamai, "The capacity region of the gaussian MIMO broadcast channel," in Proc. of IEEE ISIT, 2004.

[5] M. Sharif and B. Hassibi, "Scaling laws of sum rate using time-sharing, DPC, and beamforming for MIMO broadcast channels," in Proc. of IEEE ISIT, 2004

[6] M. Sharif and B. Hassibi "On the capacity of MIMO broadcast channel with partial side information" IEEE Trans. Info. Theory, vol. 51, no. 2 Feb. 2005.

[7] P. Viswanath, D. N. Tse, and R. Laroia, "Opportunistic beamforming using dump antennas," IEEE Trans. Inform., vol. 48, no. 6, pp. 1277 1294. June 2002

[8] H. Viswanathan and S. Venkatesan, "Asymptotics of sum rate for dirty paper coding and beamforming in multiple antenna broadcast channels," in Proc. of the 41'st Annual Allerton Conf., 2003.

[9] T. Yoo and A. Goldsmith, "On the Optimality of Multi-Antenna Broadcast Scheduling Using Zero- Forcing Beamforming" To appear: IEEE JSAC Special Issue on $4 G$ Wireless Systems.

[10] G.A. Jones, J.M. Defilippis, H. Hoffmann, and E. A. Williams, "Digital television station and network implementation," Proceedings of the IEEE, vol. 94, no. 1, pp. 22-36, Jan. 2006.

[11] U. Reimers, "Digital video broadcasting," IEEE Communications Magazine, vol. 36, no. 6, pp. 104-110, Jun. 1998.

[12] S. Pekowsky and A. Andorfer, "Multimedia data broadcasting strategies," IEEE Communications Magazine, vol. 39, no. 4, pp. 138-145, Apr. 201.

[13] A. Khisti, U. Erez, A. Lapidoth, G. Wornell, "Carbon copying onto dirty paper," submitted to IEEE Transactions. on Information Theory.

[14] Y. Steinberg and S. Shamai, "Achievable rates for the broadcast channel with states known at the transmitter," International Symposium on Information Theory (ISIT), pp. 2184-2188, Sep. 2005.

[15] A. Khitsi, Coding Techniques for Multicasting, Master's thesis, MIT Cambridge, MA, 2004.

[16] Nihar Jindal and Zhi-Quan Luo, "Capacity limites of multiple antenna multicast," International Symposium on Information Theory (ISIT), Seattle, OR, Jul. 2006.

[17] S. Vishwanath, N. Jindal, and A. Goldsmith, "Duality, achievable rates and sum rate capacity of Gaussian MIMO broadcast channel," IEEE Trans. Inform., vol. 49, no. 10, pp. 26582668, 2003.

[18] T. Y. Al-Naffouri, A. F. Dana, and B. Hassibi, "On the Capacity of Multiple Antenna Group-Broadcast Systems," in preparation for submission. 